\title{
Computational Models for Materials with Shape Memory: Towards a Systematic Description of Coupled Phenomena
}

\author{
Roderick V.N. Melnik ${ }^{1}$ and Anthony J. Roberts ${ }^{2}$ \\ 1 University of Southern Denmark, \\ Mads Clausen Institute, DK-6400, Denmark, rmelnik@mci.sdu.dk \\ 2 University of Southern Queensland, \\ Department of Mathematics and Computing, QLD 4350, Australia
}

\begin{abstract}
In this paper we propose a systematic methodology for improving computational efficiency of models describing the dynamics of materials with memory as part of multilayered structures, in particular in thermoelectric shape memory alloys actuators. The approach, based on a combination of the centre manifold technique and computer algebra, is systematic in a sense that it allows us to derive computational models with arbitrary order of accuracy with respect to certain small parameters. Computational results demonstrating the efficiency of the proposed methodology in reproducing the dynamics of austeniticmartensitic phase transformations upon thermoelectric cooling are presented.
\end{abstract}

\section{Introduction}

Coupled systems of partial differential equations arise frequently in various contexts and different areas of theory and applications. Their fundamental importance in a better understanding of complex phenomena and processes ranges from such fields as supersymmetric and string theories to fluid-structure interactions, thermoelasticity, electroelasticity, and control, to name just a few.

Models based on coupled systems of PDEs are often inheritably difficult to treat analytically and some such models are amongst the greatest computational challenges in mathematical modelling. The development of efficient computational procedures for such models lags behind the needs in their applicability in sciences and engineering. While for some such models a substantial progress has been achieved in developing efficient numerical methodologies and their rigorous justification (e.g., [10,12]), efficient computational algorithms and methodologies for many other models, especially those dealing with strongly nonlinear coupled problems, are at the beginning of their development. One problem from this class is at the main focus of the present paper.

In many engineering applications coupled phenomena are in the very essence of the successful design of systems and devices. Classical examples include electromechanical and magnetomechanical systems. As a part of modelling such systems, in some cases we also have to adequately describe the dynamic behaviour 
of complex materials these systems made of, subject to different loadings. This is known to be one of the most difficult tasks in computational sciences, which is complicated even further as soon as an intrinsic nonlinear interplay between different physical fields, such as mechanical, electric, and/or thermal, is at the heart of the process of interest. Our interest in this paper lies with the materials that under appropriate externally imposed conditions (e.g., through the action of thermal, magnetic, mechanical fields) can recover their shape after being permanently deformed. One class of such materials, known as shape memory alloys (SMA), has become increasingly important on the technological landscape, and the design of new devices and systems requires a better understanding of the dynamics of these materials, including phase transition and hysteresis phenomena [13]. The modelling of SMA dynamics is a challenging field of computational science where interdisciplinary efforts are required. To model adequately SMAbased multilayered structures represents even a greater challenge.

\section{Models for SMA-Based Multilayered Thermoelectric Devices and Their Applications}

The use of shape memory materials in microtechnological applications, where these materials have a great potential as parts of actuators and sensors, is often impeded by low dynamic responses, especially when an efficient cooling is important [1]. This is the case whenever the control of SMA phase transformations (and a subsequent mechanical power generation) is made by the temperature. Since the time constant for heat transfer is typically large compared to the small time constants required for many high frequency applications, one has to deal with the problem of low rates of cooling. While the heating is easy to implement technologically (typically, by using the Joule effect), the cooling of the SMA sample represents a more difficult task in these applications. The basic problem here is in the fact that by going to smaller scales the efficiency of traditional "cooling" technologies such as thermal convection and conduction, usually applied to SMA samples at larger scales, decreases. That is why experimentalists are looking for other technologies to overcome the problem. One of the promising technologies in this field is connected with the thermoelectric cooling based on the Peltier effect. In electronic industry this technology is used, for example, to cool electronic chips.

Thermoelectric effects have been effectively used for quite a while in power conversion and refrigeration applications, where the efficiency is usually achieved with semiconductor thermoelements. Since the efficiency of thermoelectric devices (heat engines or heat pumps) are limited by the electrical/thermal properties of the semiconductor materials (where the thermal energy is converted into electric power), much progress in the field has been connected with the development in solid-state electronics. For the benefits to the reader we recall that the use of the Peltier effect is based on the fact that whenever we apply an electric current to a system composed of two dissimilar conductors, heat is evolved at one junction and absorbed at the other, that is depending on the current direction, 
one junction becomes cold and the other becomes hot. This brings difficulties in modelling these devices at a computational level due to a jump in the heat flux at the interface caused by the Peltier effect. The basic idea pertinent to the technicalities of the device we are interested in is shown in Fig.1(a) where $J(t)$ is the current density, and $\mathrm{P}$ and $\mathrm{N}$ stand for oppositely doped semiconductors (only one thermocouple is shown in this Melcor-type design configuration). The thermoelectric process is thermodynamically reversible, and fundamentals for its modelling were laid by A.F. Ioffe (e.g., [6] and references therein). Based on those early results, semiconductors have been used for localised cooling in various applications. However, it has only recently been noted (e.g., [17, 2]) that the Peltier effect can be used for thermal cycling of the SMA. The potential of this idea in designing large strain SMA actuators have been experimentally exploited, and comparisons with other cooling mechanisms, including natural and forced convection, have been favorable. Therefore, in a way similar to Fig.1(a), the SMA actuator can be made as the cold/hot junction of a thermoelectric couple. As an example, we consider the design configuration shown in Fig. 1(b) (e.g., [2]) with the basic module given in Fig.1(c). As seen in this figure, the SMA layer is typically put in-between two semiconductor layers, usually made of bismuth telluride (TeBi). In this way, SMA can be used for actuating purposes in shape and vibration control problems, as well as in applications ranging from various mechatronics products (e.g., flexible grippers for the assembly of tiny work pieces in the semiconductor industry and the sample collection for microscopic observations in the biochemical labs) to SMA actuators in the area of underwater vehicle design, artificial valves in bioengineering applications, etc.

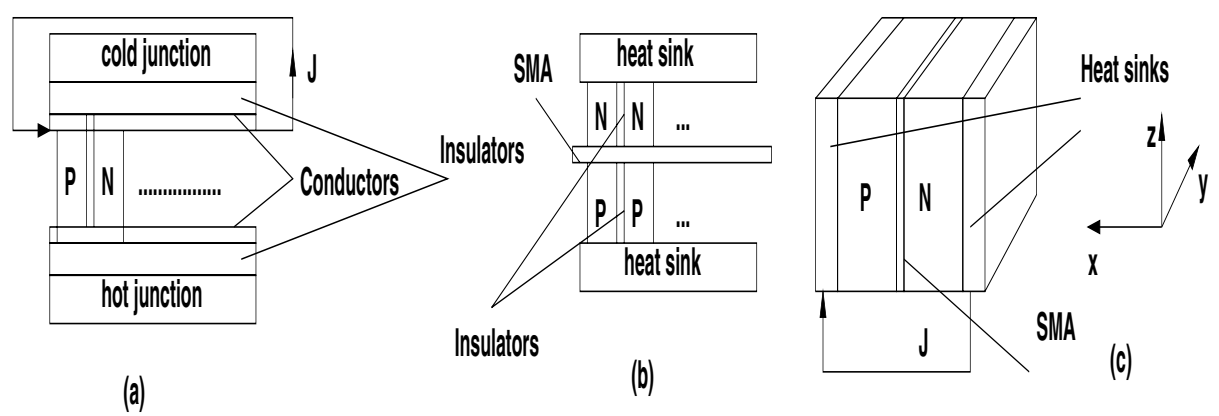

Fig. 1. Design configurations of thermoelectric devices and SMA actuator modules

The modelling of thermoelectrically cooled/heated SMA actuators is typically limited in the literature to thermal analyses only (e.g., $[1,5]$ and references therein) where the SMA module is described on the basis of the heat equations with different approximations for the internal heat productions. Such models cannot describe the dynamics of phase transitions that would require considering displacements coupled to temperature changes. In addition, until recently such models were based on the assumption of a small ratio between the SMA layer thickness and the thickness of semiconductors (see discussion in [5]). This 
assumption might be violated in practice. Nevertheless, such models allow us to obtain a first approximation to the temperature at the interface between the SMA and semiconductor layers. This fact will be used in the models discussed below. Following [4], we consider a thermal model for the multilayered SMA actuator based on a system of three heat equations written for each layer

$$
k_{i} \frac{\partial^{2} \theta_{i}}{\partial x^{2}}+\rho_{i} J^{2}(t)-H \frac{P}{A}\left(\theta_{i}-\theta_{0}\right)=C_{v}^{i} \frac{\partial \theta_{i}}{\partial t},
$$

and coupled together by the flux interface conditions, e.g.,

$$
-k_{s} \frac{\partial \theta_{s}}{\partial x}=-k_{p} \frac{\partial \theta_{p}}{\partial x}+\alpha_{p} \theta_{p} J(t) .
$$

Model (1)-(2) provides a good approximation especially in the case where the temperature in each layer does not vary significantly on the plane perpendicular to the x-axis. In (1), (2) index $i$ stands for $\mathrm{N}$ (N-type semiconductor), $\mathrm{S}$ (SMA-layer), or P (P-type semiconductor, see Fig.1). We denote the temperature by $\theta$, the thermal conductivity coefficient by $k$, the perimeter and area of the cross section by $\mathrm{P}$ and $\mathrm{A}$, respectively, the heat convection coefficient by $\mathrm{H}$, the electrical resistivity by $\rho$ (so that $\rho J^{2}$ is the Joule heat), the heat capacity per unit volume by $C_{v}$, and the Seebeck coefficient by $\alpha$. As in [9], the structure is symmetric, the thickness of the SMA layer is $a_{0}$, and the thickness of each semiconductor layer is $a$. Two other dimensions of the structure, denoted by $\mathrm{b}$ and c, are used in the definition of $A$ and $\mathrm{P}$, so that $A=b \cdot c$ and $P=2(b+c)$ (see details in [9]). By considering a special case where the thermal conductivity of SMA, $k_{s}$, is much larger compared to thermal conductivity of semiconductors, $k_{n}$, and $k_{p}$, (e.g., by assuming a small ratio between the layer thickness of SMA and semiconductor $a_{0} / a \ll 1$ ), the strong thermomechanical coupling, intrinsic to the SMA layer, has often been neglected (e.g., [4]). Although the assumption $a_{0} / a \ll 1$ (dubious in many applications) has been recently removed in [5], most approaches developed in this field so far can account only partly for the thermomechanical coupling in the SMA layer $[4,7,5]$. However, it is this coupling that is responsible for phase transformations, and due to this coupling, the often made assumption that $\theta_{s}(x, t)$ is independent of $\mathrm{x}$ (e.g., [4]) cannot be justified in any situations involving phase transformations. A partial phase transformation (and hence the effect of coupling) has been considered in [7], where the assumption of an almost uniform temperature distribution in the SMA layer has been removed. The authors of [7] used a phenomenological model based on gradual transformations of SMA polycrystals and evolution equations for field variables which were then solved with a Runge-Kutta method. Similarly to their previous works (e.g., [2]), the procedure was limited to stress-free boundaries, and therefore could be applied to a specific form of thermomechanical coupling only. A more general procedure allowing to treat different thermomechanical loadings have been considered recently in [13].

Despite limitations mentioned above, models proposed in $[4,7,5]$ are an important step forward, indicating clearly a way of reducing the problem (1), (2) to 
a relatively simple heat transfer problem with coupling effects implemented at the boundaries, as a result of the dependency of the temperature in semiconductor layers on the heat capacity of the SMA material. Indeed, as shown in [4], this problem can be effectively reduced to an integro-differential equation, which in its turn is reduced further to the solution of a Volterra equation. By considering $\theta$ as function of $\mathrm{J}$ in the SMA layer, it can be shown that certain conditions, such as bounds on J, should be satisfied in order to achieve a monotonic decay of the SMA temperature. This is an important observation since a major disadvantage in utilising SMA actuators is the low rate of cooling. However, no possible austenite-to-martensite transformations as a result of this cooling has been discussed. It is one of our purposes here to demonstrate that our computational model can reproduced very well such transformations under appropriate cooling conditions. Before proceeding with this task, thermal boundary conditions for the SMA layer should be specified.

\section{Describing Coupled Thermomechanical Fields in the SMA Layer}

In describing coupled thermomechanical fields in the SMA layer, we follow a twostep procedure. Our first step is equivalent to that described in [5]. This leads us to an approximation of the temperature on the boundary of semiconductor layers, and by using its continuity a fully coupled model for the SMA layer can be formulated. If $J(t)$ is assumed to be constant, the exact solution to the problem at step 1, considered as a "purely" thermal analysis of the multilayered structure, can be found. In this analysis, the heat transfer behaviour along the SMA layer is dominated by the temperature at the interface between the SMA layer and the semiconductor layer. The analysis can be reduced to an integrodifferential equation with respect to an auxiliary function, as discussed in [5]. More precisely, we consider equation (1) for $i=s$, that is for the SMA layer, where, due to symmetry, it is sufficient to consider the interval $0<x<a_{0} / 2$ only. This equation is supplemented by the initial condition $\theta(x, 0)=\theta_{0}$, "symmetry" boundary condition $\frac{\partial \theta}{\partial x}(0, t)=0$, and the "interface" boundary condition at $x=a_{0} / 2$ :

$$
\begin{aligned}
\frac{\partial \theta}{\partial x}= & -\left.\frac{2 k}{a k_{s}} \int_{0}^{t} G_{1}(t-\tau)\left[\frac{d \theta}{d t}+\frac{H P}{C_{v} A}\left(\theta-\theta_{0}\right)\right]\right|_{t=\tau} d \tau \\
& +\left(1-\frac{k}{\alpha a}\right) \theta+F(t), \quad t>0
\end{aligned}
$$


obtained by using the solution for the semiconductor layer, as explained in [5]. In (3) $G_{1}(t)=\sum_{n=1}^{\infty} \exp \left(-\beta_{n} t\right)$ and function $F$ has the following form:

$$
F(t)=\frac{4 \rho k}{C_{v} k_{s} a} \int_{0}^{t} \sum_{n=1}^{\infty} \exp \left(-\beta_{2 n-1}(t-\tau) J^{2}(\tau) d \tau+\frac{k}{a k_{s}} \theta_{0} .\right.
$$

Indices of the coefficients of the semiconductor adjacent to the SMA layer are omitted in (3) and (4), and $\beta_{n}=n^{2} \pi^{2}+H$. It is this problem that is reduced to an integro-differential equation. Existence and uniqueness of its solution is established in a standard manner by equivalence with a Volterra equation, and its solution is found numerically by employing a finite difference scheme. Temperature profiles at the interface between the SMA and semiconductor layers can be quite different for different values of the current density $\mathrm{J}$, but once such a profile is found we can proceed to the second step of our procedure.

At the second step, we study the fully coupled thermomechanical dynamic system of the SMA layer by employing a general procedure, similar to that discussed in [13] for a single layer structure. Starting from the 3D coupled model consisting of the equation of motion and the energy balance equation

$$
\left\{\begin{array}{l}
\rho \frac{\partial^{2} \mathbf{u}}{\partial t^{2}}=\nabla \cdot \mathbf{s}+\mathbf{F}, \\
\rho \frac{\partial e}{\partial t}+\rho \tau_{0} \frac{\partial^{2} e}{\partial t^{2}}-\mathbf{s}^{T}:(\nabla \mathbf{v})-\tau_{0} \frac{\partial}{\partial t}\left[\mathbf{s}^{T}:(\nabla \mathbf{v})\right]-\nabla \cdot(k \nabla \theta)=G,
\end{array}\right.
$$

we aim at developing efficient computational models allowing to reproduce austeniteto-martensite phase transformations observed under thermoelectric cooling of SMA actuators. In (5) $\mathbf{u}$ denotes displacements, $\mathbf{s}$ is the stress tensor, $\mathbf{v}=\partial \mathbf{u} / \partial t$ is the velocity vector, $e$ is the internal energy, $\tau_{0}$ is the relaxation time, $\mathbf{F}$ and $G$ are forcing terms (further details can be found in [8]). Constitutive models used in this paper are based on the general representation of the free energy function in the form

$$
\Psi(\boldsymbol{\epsilon})=\psi^{0}(\theta)+\sum_{i=1}^{\infty} \sum_{j=1}^{j^{i}} \psi_{j}^{i} \mathcal{I}_{j}^{i}
$$

with $\mathcal{I}_{i}^{j}$ being strain invariants, $\psi_{i}^{j}$ being temperature dependent functional coefficients, and $\boldsymbol{\epsilon}$ being the strain tensor. Function (6) is made invariant with respect to the symmetry group of austenite, and the upper limits $j^{i}$ are chosen appropriately to satisfy this condition [8]. Further, we determine the stress component due to mechanical dissipations as $\mathbf{s}^{q}=\rho \frac{\partial \Psi}{\partial \boldsymbol{\epsilon}}$. Thermal dissipations, plasticity, and other effects can be incorporated into the model, but in what follows we describe our methodology for the Landau-Devonshire type constitutive models as an example, where we take $\mathbf{s}=\mathbf{s}^{q}$. In particular, for the general 3D model we use the Falk-Konopka representation of the free energy function (6) 
with 10 strain invariant directions, valid for the copper-based SMA materials [8].

\section{Reducing Computational Complexity and Preserving Essential Properties of the System with Centre Manifold Models}

Model (5), supplemented by appropriate constitutive laws for the SMA materials, boundary and initial conditions, represents a tremendous computational challenge in the general 3D case. Any computational treatment of this model aiming at the description of the dynamics of SMA-based system responses will necessarily require essential simplifications of this model in order to be tractable. Such simplifications should be developed in a systematic way, and below we propose a systematic methodology for improving computational models for the description of SMA dynamics. Our idea is based a combination of the centre manifold technique and computer algebra for developing reduction procedures for the original model (5) on centre manifolds, while retaining essential properties of the system. Such procedures have been used successfully in a number of applications $[15,18,16]$, and have recently received an increasing interest in the context of computations of normal forms, an apparently useful methodology in the analysis of nonlinear oscillations.

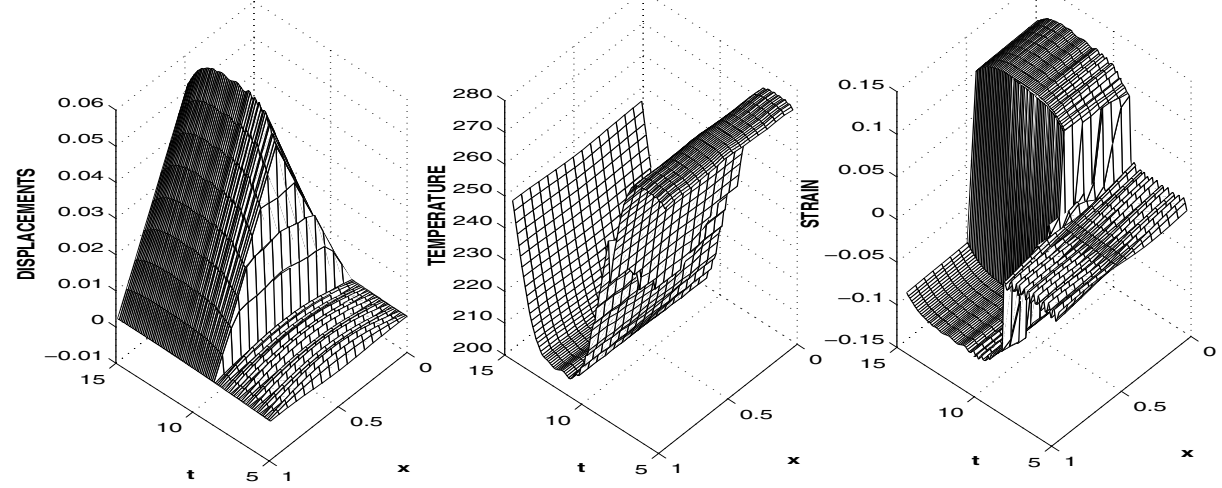

Fig. 2. Austenite-to-martensite phase transformation in the SMA layer of the thermoelectric actuator (from left to right): (a) displacements, (b) temperature, (c) strain.

A quite general theoretical result has been obtained in [3] confirming the existence of an invariant manifold (called a centre manifold) of the invariant set which contains every locally bounded solution. The importance and the need for developing new algorithms in theory and applications of approximation dynamics has been recently re-emphased in a fundamental work [14]. In a series of papers $[8,9,13]$ we have initiated applications of the centre manifold technique to studying complex nonlinear coupled systems describing the behaviour 
of shape memory alloys, and in this paper we apply this technique to modelling multilayered structures such as those depicted in Fig. 1.

Our approach allows considering different patterns of thermomechanical coupling implemented via appropriate choices of the free energy function, constitutive models, initial and boundary conditions. In the dimensions higher than one computational results are virtually absent in this field, and little is know on how to simplify such models as (5), (6) systematically. In the spirit of our previous works $[8,13]$ we consider a shape memory alloy slab, and associate the qualitative change in system behaviour with the subset of eigenvalues having zero real parts. Then, critical variables are chosen as those that are responsible for the essential behaviour of the system. This leads to the following steps in our computational procedure:

- Analysis of eigenvalues of the cross-slab modes, as required by centre manifold theory;

- Construction of a sub-center manifold based upon the relevant eigenmodes as they evolve slowly.

The low-dimensional invariant manifold should be parametrised by the amplitudes of the critical modes, which constitute a subset of all modes, and determine the essential dynamic behaviour of the system [15]. A computational model is constructed with respect to these amplitudes, and since the leading order structure of the critical eigenmodes are constant across the slab (the longitudinal variations were neglected in the first approximation), we associate these amplitudes with the y-averages of displacements, velocities, and temperature, denoted here as $\mathbf{U}=\left(U_{1}, U_{2}\right), \mathbf{V}=\left(V_{1}, V_{2}\right)$, and $\theta^{\prime}=\theta-\theta_{0}\left(\theta_{0}\right.$ is taken as $\left.300^{\circ} \mathrm{K}\right)$. The connection between these variables, and the variables of the original model is established in an approximate form by employing the low-dimensional invariant manifold and asymptotic sum representations (explicit formal power series representations maybe prohibited even for a computer). Similar to multiple scale techniques, this approach requires a balancing of the order of small effects in the construction of the model, not in its use [16]. The small parameters used in this paper are $\partial_{x}, E=\left\|\boldsymbol{U}_{x}\right\|+\left\|\boldsymbol{V}_{x}\right\|$ and $\vartheta=\left\|\Theta^{\prime}\right\|$, and the computer algebra program has been written to balance their small effects. We treat the strains as small, as measured by $E$, while permitting asymptotically large displacements and velocities. It is important to emphasise that such approximations can be derived up to arbitrary order of accuracy, as explained in [8]. For the model discussed below, the approximations were derived with errors $\mathcal{O}\left(E^{5}+\partial_{x}^{5 / 2}+\vartheta^{5 / 2}\right)$ where the notation $\mathcal{O}\left(E^{p}+\partial_{x}^{q}+\vartheta^{r}\right)$ is used to denote terms involving $\partial_{x}^{\beta_{2}} E^{\beta_{1}} \vartheta^{\beta_{3}}$ such that $\beta_{1} / p+\beta_{2} / q+\beta_{3} / r \geq 1$. In summary, after the decision on how to parametrise the centre manifold model is taken, and the critical modes are identified in order to project the dynamics onto the "slow" modes of interest, we complete our computational procedure by

- Substituting the asymptotic sums into the governing equations;

- Evaluating residuals by using computer algebra tools to get the result with the required accuracy. 
These steps can be performed in a computationally very efficient manner by using an iterative algorithm analogous to that developed in [15]. By applying the above procedure, we have derive the reduced model

$$
\left\{\begin{array}{l}
\rho \frac{\partial V_{1}}{\partial t}=\frac{\partial s}{\partial x}+F, \quad \frac{\partial U_{1}}{\partial t}=V_{1} \\
C_{v} \frac{\partial \Theta^{\prime}}{\partial t}=k \frac{\partial^{2} \Theta^{\prime}}{\partial x^{2}}+\left(c_{11}+c_{12} \Theta^{\prime}-c_{13}\left(\Theta^{\prime}\right)^{2}\right) \frac{\partial U_{1}}{\partial x} \frac{\partial V_{1}}{\partial x}+ \\
\quad+\left(c_{14}+c_{15} \theta^{\prime}\right) \frac{\partial V_{1}}{\partial x}\left(\frac{\partial U_{1}}{\partial x}\right)^{3}+c_{18} \frac{\partial V_{1}}{\partial x}\left(\frac{\partial U_{1}}{\partial x}\right)^{5}+g \\
s=\left(c_{1}+c_{2} \theta^{\prime}-c_{3}\left(\theta^{\prime}\right)^{2}\right) \frac{\partial U_{1}}{\partial x}-\left(c_{4}-c_{5} \theta^{\prime}\right)\left(\frac{\partial U_{1}}{\partial x}\right)^{3}+c_{6}\left(\frac{\partial U_{1}}{\partial x}\right)^{5}
\end{array},\right.
$$

where coefficients $c_{k}$ are positive material constants (taken here as in [8]). This model, derived from the general 3D model (5), is exact up to the 4th order with respect to the small parameters, and preserves all essential features of the dynamics of the original system. In the case analysed here, if dissipations are omitted, there is a zero eigenvalue of multiplicity 5 and the rest are purely imaginary. Therefore, the sub-centre manifold has been constructed based on these 5 eigenmodes. Note that for simplicity we consider here the critical eigenvalues that are zeros (see comments on pure imaginary eigenvalues in $[15,16]$ ). The initial conditions for the detailed (original) dynamics also have to be projected onto the low-dimensional manifold, as well as the boundary conditions (see further details on this in $[8,13])$. The resulting model $(7)$ is solved by its reduction to a system of differential-algebraic equations, and the displacements and temperature of the slab are recovered by using critical eigenmodes.

The developed computational procedure has been applied to a number of problems dealing with multilayered SMA actuators. Fig. 2 provides one of the results based on this procedure. Profiles for thermal cycling used in these experiments are analogous to those used in thermal analyses of these devices [4, 5]. Starting with high temperature phase (austenite) (represented in Fig. 2(a) and 2(c) by zero displacements and zero strain, respectively) it is shown how the cooling of the SMA layer (Fig. 2(b)) leads to the martensitic phase. Other results of computational experiments demonstrating the efficiency of the centremanifold-based procedures in capturing all main features of macroscopic phase transitions can be found in $[9,8,13]$.

Using our approach it is possible to construct a hierarchy of mathematical models for the description of the dynamics of shape memory alloys. Computational efforts spent for deriving centre manifold models are reduced substantially by employing computer algebra to deal with asymptotic sums in several small parameters chosen to measure the influence of small effects [15]. Since such centre manifold reductions can be derived with arbitrary degree of accuracy, this approach is systematic and deserves more attention in computational science community. With further advances in microtechnologies, for such multilayered 
structures as discussed in this paper more refined models for semiconductor layers will also be required. Such models by themselves represent a difficult and challenging task in computational sciences (see [11]).

\section{References}

1. Abadie, J., Chaillet, N., Lexcellent, C., Bourjault, A.: Thermoelectric Control of SMA Microactuators: a Thermal Model. SPIE Proceedings. 3667 (1999) 326-336

2. Bhattacharyya, A., Lagoudas, D.C., Thermoelectric SMA Actuators and the Issue of Thermomechanical Coupling. J. Phys. IV. 7 C5 (1997) 673-678

3. Chow, S.-N., Liu, W., Yi, Y.: Center Manifolds for Invariant Sets. J. of Diff. Equations. 168 (2000) 355-385

4. Ding, Z., Lagoudas, D.C.: Solution Behavior of the Transient Heat Transfer Problem in Thermoelectric SMA Actuators. SIAM J. of Appl. Math. 57 (1997) 34-52

5. Ding, Z., Lagoudas, D.C.: Transient Heat Transfer Behaviour of 1D Symmetric Thermoelectric SMA Actuators. Math. and Computer Modelling. 29 (1999) 33-55

6. Egli, P.H. (ed.): Thermoelectricity. Wiley, New York \& London (1960)

7. Lagoudas, D.C., Ding, Z.: Modeling of Thin Layer Extensional Thermoelectric SMA Actuators. Int. J. Solids Structures. 35 (1998) 331-362

8. Melnik, R.V.N., Roberts, A.J., and Thomas, K.A.: Computing Dynamics of CopperBased SMA via Centre Manifold Reduction of 3D Models. Computational Materials Science. 18 (2000) 255-268

9. Melnik, R.V.N. and Roberts, A.J.: Modeling dynamics of multilayered SMA actuators. SPIE Proceedings. 4235 (2000) 117-125

10. Melnik, R.V.N.: Generalised Solutions, Discrete Models and Energy Estimates for a 2D Problem of Coupled Field Theory. Applied Mathematics and Computation. 107 (2000) 27-55

11. Melnik, R.V.N., He, H.: Modelling Nonlocal Processes in Semiconductor Devices with Exponential Difference Schemes. J. of Engineering Mathematics. 38 (2000) 233-263

12. Melnik, R.V.N.: Discrete Models of Coupled Dynamic Thermoelasticity for StressTemperature Formulations. Applied Mathematics and Computation. 122 (2001) $107-132$

13. Melnik, R.V.N., Roberts, A.J., and Thomas, K.A.: Coupled Thermomechanical Dynamics of Phase Transitions in Shape Memory Alloys and Related Hysteresis Phenomena. Mechanics Research Communications. (2001) to appear

14. Pliss, V.A., Sell, G.R.: Approximation Dynamics and the Stability of Invariant Sets. J. of Diff. Equations. 149 (1998) 1-51

15. Roberts, A.J.: Low-Dimensional Modelling of Dynamics via Computer Algebra. Computer Physics Communications 100 (1997) 215-230

16. Roberts, A.J.: Computer Algebra Derives Correct Initial Conditions for LowDimensional Dynamical Systems. Computer Physics Communications 126 (200) 187-206

17. Shanin, A.R., Meckl, P.H., Jones, J.D., Thrashner, M.A., Enhanced Cooling of SMA Wires Using Semiconductor "Heat Pump" Modules. J. of Intell. Material Systems and Structures. 5 (1994) 95-104

18. Thomsen J.J.: Vibrations and Stability. Order and Chaos. McGraw-Hill, London (1997) 